\title{
Towards a comprehensive catalog of human genes associated with main forms of pathozoospermia and its functional annotation
}

\author{
Elena V. Ignatieva \\ Institute of Cytology and Genetics, \\ SB RAS \\ Novosibirsk, Russia \\ eignat@bionet.nsc.ru \\ Ludmila V. Osadchuk \\ Institute of Cytology and Genetics, \\ SB RAS \\ Novosibirsk, Russia \\ losadch@bionet.nsc.ru
}

\author{
Alexander V. Osadchuk \\ Institute of Cytology and Genetics, \\ SB RAS \\ Novosibirsk, Russia \\ osadchuk@bionet.nsc.ru;
}

\author{
Maxim A. Kleshev \\ Institute of Cytology and Genetics, \\ SB RAS \\ Novosibirsk, Russia \\ max82c11@bionet.nsc.ru
}

\begin{abstract}
The genetic causes of the global decline in male fertility is among the hot spots of scientific research in reproductive genetics. The most common way to evaluate male fertility in clinical trials is to determine the quality of the ejaculate. A pathological condition characterized by lower quality indicators of the ejaculate compared to the norm and leading to impaired fertility is called pathozoospermia. Pathozoospermia is a syndrome that occurs in many diseases and can be caused by many factors, including genetic ones. To systematize information about genes associated with main forms of pathozoospermia, we created a catalog of such genes and analyzed their functional characteristics. On the basis of a manual analysis of scientific publications we collected data on $\mathbf{7 0}$ genes harboring more than one hundred allelic variants associated with pathozoospermia. Gene ontology enrichment analysis revealed that genes were significantly associated with spermatogenesis and male gamete generation. In addition it was found that genes from the catalog were associated with more than seventy human diseases that were both reproductive system pathologies and others, such as Parkinson's and Alzheimer's diseases as well as various cancer types.
\end{abstract}

Keywords - male infertility, spermatogenesis, pathozoospermia, genetic polymorphism, associations, database, functional annotation

\section{Introduction}

The decline in male reproductive potential, identified in many countries of the world, is an important scientific problem and serious social issue $[4,5]$. The question of the genetic causes of the global decline in male fertility is among the hot spots of scientific research in reproductive genetics. It is believed that in $20-25 \%$ of cases of severe reproductive dysfunction in men can be due to genetic causes [4]. Such a high frequency of genetic disorders of male reproductive function indicates its multigenic control. The most common way to evaluate male fertility in clinical trials is to determine the quality of the ejaculate. For this purpose, a number of quantitative indicators are used, defined as the reference values of WHO [6]. A pathological condition characterized by lower quality indicators of the ejaculate compared to the norm and leading to impaired fertility is called pathozoospermia. The most common forms of pathozoospermia are azoospermia, oligozoospermia, asthenozoospermia teratozoospermia. Pathozoospermia is a syndrome that occurs in many diseases and can be caused by many factors, including genetic ones. Despite the urgency of the problem of male infertility, there is currently no specialized information resource accumulating information about the genes associated with specific forms of pathozoospermia. These data are dispersed in a large number of scientific publications, and such database on associations between genes and diseases as ClinVar, OMIM and DiGeNet, contain data by no means on all forms of pathozoospermia. To systematize information about genes associated with main forms of pathozoospermia, we created a catalog of such genes and analyzed their functional characteristics.

\section{Materials and Methods}

Data on associations between allelic variants of genes and specific forms of pathozoospermia were extracted from scientific publications that were selected using ANDSystem [3]. Search terms that refer to specific forms of pathozoospermia were obtained from [6]. Gene ontology (GO) and human disease ontology enrichment analysis were performed using DAVID tool [2]. The enriched GO terms $(\mathrm{FDR}<0.05)$ from the biological processes vocabulary GOTERM_BP 5 and enriched diseases $(F D R<0.05)$ from the genetic association database [1] were considered in our study.

\section{Results}

First we formed a following dictionary of keywords denoting specific forms of pathozoospermia: (1) azoospermia; (2) non-obstructive azoospermia; (3) cryptozoospermia; (4) oligozoospermia; (5) severe oligozoospermia; asthenozoospermia; (7) teratozoospermia; oligoasthenoteratozoospermia (OAT); oligoasthenozoospermia; (10) oligoteratozoospermia; (11) globozoospermia. Using these key words as a search terms we collected data on 70 genes harboring more than one hundred allelic variants associated with pathozoospermia. The largest portions of genes were associated with oligozoospermia and non-obstructive azoospermia (Fig.1A). Most genes (63 out of 70) were located on autosomes, $\mathrm{X}$ and $\mathrm{Y}$ chromosomes had three genes each, and one gene was located on mitochondrial DNA (Fig. 1B). encoded transcription factors: WT1, AHR, 


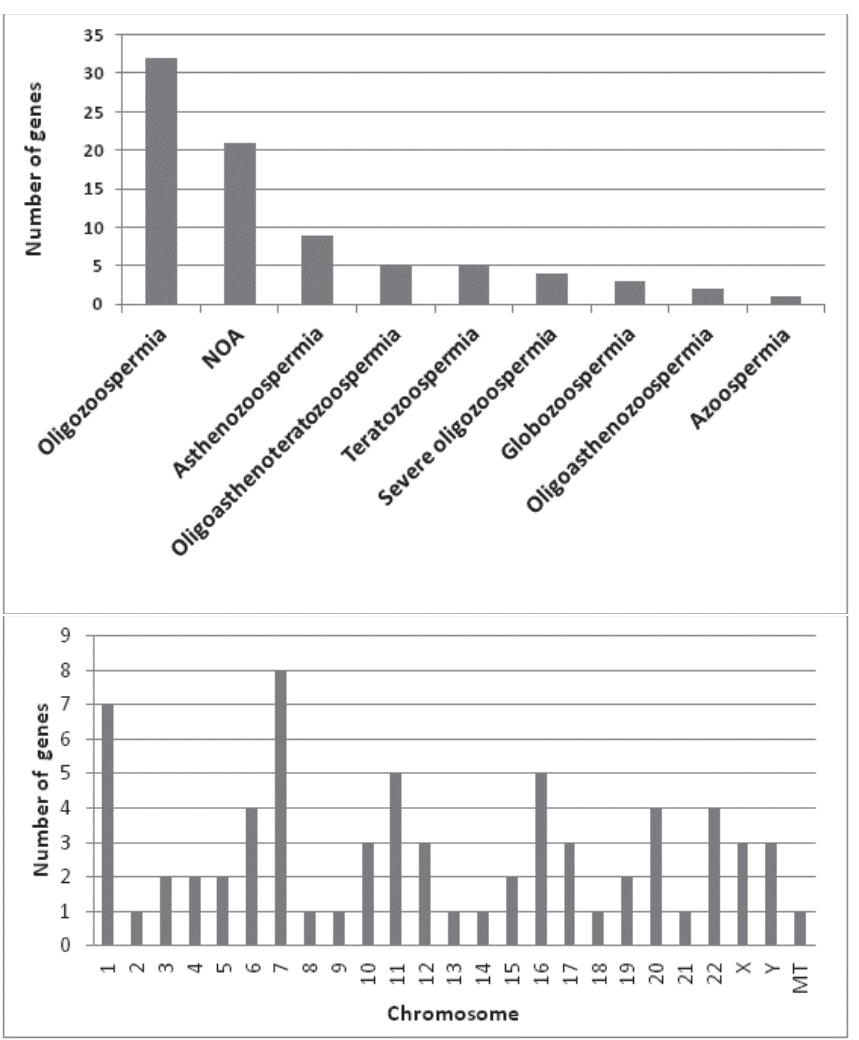

Fig. 1. Information content of the catalog. A - the number of genes associated with a specific form of pathozoospermia; B - the number of genes from the catalog identified on each chromosome. NOA non-obstructive azoospermia; MT - non-nuclear(mitochondrial)

Enrichment analysis performed using the DAVID-tool revealed 17 significantly enriched $(\mathrm{FDR}<0.05)$ biological processes from the GOTERM BP 5 vocabulary. The most representative gene groups (more thān seven genes each) were annotated by enriched terms associated with spermatogenesis and male gamete generation (Table 1). In addition we identified that genes from the catalog were associated with more than seventy human diseases from [1] (data not shown). Among these diseases were both reproductive system pathologies and others, such as Parkinson's and Alzheimer's diseases as well as various cancer types.

\section{Conclusion}

We have created a catalog of genes and their allelic variants associated with various forms of pathozoospermia. This information was obtained on the basis of a manual analysis of scientific publications, which indicates a high level of reliability. The data we have accumulated and the results of functional gene annotation can be useful both for interpreting the results of genome- and exome-wide association studies, as well as for developing new approaches to the diagnosis and treatment of male infertility.

\section{ACKNOWLEDGMENT}

This study was supported by the grant from the Russian Science Foundation (project No. 19-15-00075).

\section{REFERENCES}

[1] K.G. Becker, K.C. Barnes, T.J. Bright, S.A. Wang, "The genetic association database", Nat Genet, Vol. 36(5), pp. 431-432, May 2004.

[2] D.W. Huang, B.T. Sherman, Q. Tan, J.R. Collins, W.G. Alvord, J. Roayaei, R. et al., "The DAVID Gene Functional Classification Tool: a novel biological module-centric algorithm to functionally analyze large gene lists", Genome Biol, Vol. 8(9), p. R183, 2007.

[3] V.A. Ivanisenko, P.S. Demenkov, T.V. Ivanisenko, E.L. Mishchenko, O.V. Saik, "A new version of the ANDSystem tool for automatic extraction of knowledge from scientific publications with expanded functionality for reconstruction of associative gene networks by considering tissue-specific gene expression", BMC Bioinformatics, Vol. 20(Suppl 1), p. 34, 2019.

[4] C. Krausz, F. Cioppi, A. Riera-Escamilla, "Testing for genetic contributions to infertility: potential clinical impact", Expert Rev Mol Diagn, Vol. 18(4), pp. 331-346, 2018.

[5] C. Krausz, A. Riera-Escamilla, "Genetics of male infertility", Nat Rev Urol, Vol. 15(6), pp. 369-384, 2018.

[6] WHO, "WHO laboratory manual for the examination and processing of human semen", 5-th edition, Cambridge Univer. Press. 2010. https://apps.who.int/iris/bitstream/handle/10665/44261/978924154778 9 _eng.pdf

TABLE 1. OVERREPRESENTED GO TERMS THAT CHARACTERIZE GENES FROM THE CATALOG

\begin{tabular}{|c|c|c|c|}
\hline Term $^{\text {a }}$ & $\begin{array}{c}\text { Number of } \\
\text { genes }\end{array}$ & $\begin{array}{c}\text { Fold } \\
\text { Enrichment }\end{array}$ & FDR \\
\hline Gamete generation & 25 & 10.5 & $1.5 \mathrm{E}-15$ \\
\hline Cell development & 22 & 3.0 & $6.8 \mathrm{E}-3$ \\
\hline Spermatogenesis & 21 & 11.0 & $1.1 \mathrm{E}-12$ \\
\hline Male gamete generation & 21 & 11.0 & $1.1 \mathrm{E}-12$ \\
\hline Germ cell development & 15 & 17.2 & $1.6 \mathrm{E}-10$ \\
\hline Gonad development & 9 & 11.2 & $1.9 \mathrm{E}-3$ \\
\hline $\begin{array}{c}\text { Development of primary } \\
\text { sexual characteristics }\end{array}$ & 9 & 10.9 & $2.3 \mathrm{E}-3$ \\
\hline Spermatid development & 8 & 16.3 & $8.4 \mathrm{E}-04$ \\
\hline Spermatid differentiation & 8 & 15.7 & $1.1 \mathrm{E}-3$ \\
\hline
\end{tabular}

a. Terms associated with more than 7 genes are presented 